\title{
How to Use Software Restoration and Virtual Computer Technology in Computer Experiment Teaching
}

\author{
He Lin \\ Langfang Polytechnic Institute, Langfang, 065000, China
}

Keywords: Computer, Experiment teaching, Software restoration

\begin{abstract}
With the progress of science and technology, the computer experiment teaching model has been promoted, verifying test has been decreasing, and computer teaching experiment plays an important role in teaching. Currently, for the shortcomings and limitations in the computer experiment teaching, a reasonable solution is proposed, and virtual computer technology and software restoration technology are used to construct a teaching environment, appropriately develop more experiment teaching models and reasonably change the environment, so as to improve the work efficiency. This paper mainly analyzes how to apply software restoration and virtual computer technology in computer experiment teaching.
\end{abstract}

\section{Introduction}

In the process of computer experiment teaching in universities, it is required to pay high attention to the cultivation of the students' practical operation ability, not only concern the interpretation of computer theory knowledge, but also use experiment teaching method to make the students apply theoretical knowledge in practice reasonably. However, there exist certain shortcomings in the computer experiment teaching of some universities in China. For example, there are no sufficient operation conditions, making it unable to construct an experiment environment, therefore, some experiments cannot be conducted normally. Many experiments need to use a lot of network equipment and more computers, but due to limitation in condition, the students cannot conduct experiment; a specific environment might be needed during teaching, but reconfiguration and reinstallation are needed each time, which will cost a lot of time, human resources and materials. For the existing limitation and problems, virtual computer software and technology are used to scientifically restore the experiment teaching to fully meet the actual demand for the computer experiment teaching, thus creating a good environment for the students.

\section{Reasonably design computer experiment teaching environment construction plan}

\section{Apply software restoration technology}

The main purpose to reasonably restore the computer system is to restore the normal system work status under the condition of not reinstalling the system and not damaging the raw data of system. Currently, there are many types of system restoration software, but the most appropriate in computer experiment teaching is Deep Freeze restoration software. As the system restoration software developed by Faronics, this software can automatically restore the system to the original state, thus ensuring that the system will not be changed and can effectively defend conscious or unconscious artificial destruction and virus invasion. The installation of Deep Freeze will not influence the operating system and hardware partition, and it also has the advantages of dynamic protection, simple operation, convenient installation and transient recovery. After installing Deep Freeze restoration software in the system whether to delete files or install files or change the system, the initial computer application condition will be recovered just by restarting the computer. Generally, Deep Freeze restoration software can be mainly divided into two forms, namely standalone version and relatively complicated enterprise version, when installing the standalone restoration software, it is required to install it one by one in sequence, making it very difficult for management, and this method is more appropriate for the computer without unified management and network link. The server version of Deep Freeze restoration software makes up and improves the above disadvantages, and it can manage 
the client in a unified manner by using C/S. Deep Freeze occupies relatively small resources and is convenient to use, and in case of system fault, it can also initialize the system appropriately ${ }^{[1]}$.

\section{Virtual computer technology}

Virtual computer is actually a relatively improved system function formed with in software simulation manner, which can smoothly operate the computer system in a completely isolated space. With the virtual software, the simulation of more virtual computers can be completed by setting a physical computer accordingly, and it is possible to access the network resources with relevant software and applications on the virtual computer, which is consistent with the real computer operation method. For the programs that can operate on the virtual computer, virtual computer is actually a real computer. For the users, virtual computer belongs to a computer application of physical operation. Once in case of breakdown of virtual computer during system evaluation of relevant software it is possibly to use the system recovery function, so as to restore the system to the safe software stage. At present, the popular and widely used virtual computers in the market mainly include Virtual PC, VMware and Virtual Box. VMware Company has researched VMware Workstation virtual computer software, which can provide the users with different system operations in a single desktop, and it is also possible to test the system, develop and deploy specific software and application. VMware Workstation can make the users conduct more different operations simultaneously in the same computer ${ }^{[2]}$.

\section{Organically combine more technologies to construct an experiment teaching environment}

Take a single laboratory as the basic example, virtual computer technology and restoration software and other relevant technologies are used to form an easy-to-operate, safe and reliable computer experiment teaching environment that can adapt to more teaching environments. The basic operation steps are:

First, install a Windows system needed for the computer experiment appropriately in the computer Client, and reasonably optimize relevant systems and appropriately install the relatively common tool software in experiment appropriately. For the virtual computer software VMware Workstation and experiment-related software, reasonably copy the created virtual computer file to the hard disk.

Second, reasonably copy the system information on the Client to the Server reasonably.

Third, Install Deep Freeze enterprise version appropriately on the Server. After installation, use software to customize the client management program and form seed file, and in addition, it is also possible to install electronic classroom program appropriately.

Fourth, install the seed computer on the computer Client, and it is also possible to install advanced electronic classroom client appropriately.

Fifth, clone relevant systems on the computer Clint to other computers for the experiment research with clone software system for convenience of normally and orderly carrying out work in the later stage.

Sixth, in the computer Server, apply the Deep Freeze enterprise version network control platform to reinstall the corresponding Deep Freeze client for the computer to which the seed file for installation has been completed. After installation, it is required to restart the computer, and the system will enter the safety protection state automatically to scientifically protect relevant client systems.

Seventh, complete the construction of computer laboratory teaching environment. Teachers can use relevant electronic classroom of computer Server in teaching practice, while students can operate relevant operating systems in the computer Client, so as to open the VMware Workstation virtual teaching system.

Through the teaching methods above, we can rapidly construct a laboratory teaching environment with strong appropriateness rapidly. On the basis of being protected by the Deep Freeze system software, many relevant experiments are completed, and the students also can participate in experiment freely in this process. Once in case of system problems, it is possible to restart the computer to restore the system. For some experiments that cannot be conducted in the current environment, it is possible to appropriately open the virtual system for experiment. 


\section{Apply virtual computer technology in computer experiment teaching Operating system experiment}

Computer has been popularized in recent years' economic development, and its application in teaching lays its unshakable status. At present, Windows system is generally used in the computer laboratory teaching of China, and influenced by the system nature, it is unable to complete the practical operation of Unix, Linux and other laboratory teaching. If any computer is installed with more operating systems, it will be inevitably to cause the later maintenance difficulty and risk problems of computer. In the Windows operating system, it is possible to appropriately introduce relevant virtual technologies, scientifically install and set Unix, Linux and many other types of operating systems, and then operate more operating systems through the virtual computer software, and it is possible to switch and operate more operation programs without secondary starting of computer $^{[3]}$. The students at present can construct an independent virtual computer in laboratory by fully applying the virtual computer technology, and can provide each type of virtual computer with independent hard disk, CMOS and relevant operating systems, thus providing convenience for the installation of formatting and applied software, and it is possible to modify the registry or configure basic files, and also conduct some destructive tests, without worrying about the damage of virtual computer or operating systems. Therefore, any problems occurring can be deleted, copying can be conducted through virtual computer, and then restart the computer to complete operation.

\section{Maintenance experiment and network construction}

The computer network laboratory needs more network connection equipment and more computers, but the virtual computer technology can complete the simulation of more computers just by using one independent computer, and it is also possible to simulate relevant network card, switch and other facilities, so as to be convenient to fully conform to the demand for network construction. In the virtual network, students can establish a domain or peer-to-peer network-based LAN under the precondition of work group ${ }^{[4]}$. It is possible to use the advantage of virtual computer of deleting hardware at will to delete and reduce the hard disk on the virtual computer, so as to form a disk-free network environment. So the teachers are required for pretreatment before experiment, for example, prepare the virtual computer and virtual network required for the computer experiment teaching, transmit relevant data of virtual computer and virtual network more rapidly to the server for sharing, so as to carry out various experiments more rapidly, including network setting and connectivity test.

\section{Network safety test}

The continuously popularizing and developing network technology has brought many conveniences to use, but also has accelerated the R\&D and spreading of computer virus, so the network safety education also has been gradually concerned by the society. However, due to the restriction of teaching conditions and environment, in many times, the teachers just stay on the theoretical level when interpreting network virus-related theoretical knowledge for students. If real demonstration is conducted during the explanation of computer virus, the teaching equipment will be damaged, and even will not work normally. Through the virtual computer technology, the virus file can be reasonably implanted in the virtual system, so as to demonstrate the principles of spreading of worm, Trojan and other virus programs, and the specific measures to defending these viruses, and ask the students for practical operation.

\section{Software test deterioration programming experiment}

In the process of Web programming experiment, we need to coordinate the browser end and server end to complete the programming of the server end and browser end in the programming link. For the students to fully understand and master the basic demand for the system development environment configuration, for example, install Apache server which can well support the access reaching of PHP, ASP and JSP etc. and set the user access number, in the process of executing database experiment, using the virtual system, students can reasonably install and use My SQL, Microsoft SQL Server and other different databases, and when studying the program and network design-related courses, 
students need to compile programs under various different environments and conduct the corresponding test in many operating systems, the test environments mainly include standalone environment, network environment and multi-version browser environment, and the main operating systems used include Unix, Linux and Windows etc. During the design of computer experiment teaching, virtual computer related technology is reasonably used to effective ensure that the students can test various software in the virtual system. Only in this way will be it able to find out and solve the problems existing.

\section{Establish a specific environment through virtual computer}

During the computer experiment teaching, sometimes specific software teaching environment is needed, but not each kind of computer system can provide the most appropriate teaching environment for the experiment teaching. If the computer equipment is updated, it is required to reinstall various software in the system, which will bring great burden to the experiment teaching.

However, in the process of experiment teaching, if it is able to properly apply the virtual computer technology, it will be able to solve the difficulties above very rapidly, and the process is also very simple. As long as the software required for the experiment teaching is installed in the virtual computer system, when it is needed to change the equipment, whether the computer owns the environmental basis to operate the software, it is only needed to safely operate the virtual computer to achieve the purpose.

\section{Conclusion}

As stated above, optimizing the teaching environment can well reflect the function of resources, so as to solve the problems of high investment cost and insufficient equipment etc., and form a relatively meticulous and complicated network and experiment environment on this basis, so as to correspond to the actual demand of experiment teaching, ensure that the whole teaching can proceed smoothly and strengthen the reasonableness of experiment result. In addition, it is also possible to reduce the damage of system components or the problem of system breakdown, which is of great help to the overall system management and maintenance. Such combined technology has brought opportunities and platforms to the computer experiment teaching, with a very good application prospect.

\section{References}

[1] Li Hongru, Application of Virtual Technology in Computer Experiment Teaching, Experiment Technology and Management, 2010, 27(5):90-92.

[2] He Zengying, Chen Jianru, Virtual Technology-based Computer Experiment Teaching, Experiment Technology and Management, 2012,29(1):79-82.

[3] Huang Xiaohua, Application of Software Restoration and Virtual Computer Technology in Computer Experiment Teaching, Software Guide, 2014(10):194-196.

[4] Gu Feng, Research on Application of Virtual Computer Technology in Computer Experiment Teaching, China Science and Technology Information, 2011(4):232-233,238. 